\title{
Multipotent adult progenitor cells from bone marrow differentiate into functional hepatocyte-like cells
}

\author{
Robert E. Schwartz, ${ }^{1}$ Morayma Reyes, ${ }^{1}$ Lisa Koodie, ${ }^{1}$ Yuehua Jiang, ${ }^{1}$ Mark Blackstad, ${ }^{1}$ \\ Troy Lund, ${ }^{1}$ Todd Lenvik, ${ }^{1}$ Sandra Johnson, ${ }^{1}$ Wei-Shou Hu, ${ }^{2}$ and Catherine M. Verfaillie ${ }^{1,3}$
}

\author{
${ }^{1}$ Stem Cell Institute, \\ ${ }^{2}$ Department of Chemical Engineering, and \\ ${ }^{3}$ Division of Hematology, Department of Medicine, University of Minnesota, Minneapolis, Minnesota, USA
}

Address correspondence to: Catherine M. Verfaillie, University of Minnesota,

Mayo Mail Code 716, 422 Delaware Street SE, Minneapolis, Minnesota 55455, USA.

Phone: (612) 625-0602; Fax: (612) 624-2436; E-mail: verfa001@tc.umn.edu.

Robert E. Schwartz and Morayma Reyes contributed equally to this work.

Received for publication February 2, 2002, and accepted in revised form April 2, 2002.

\begin{abstract}
We have derived from normal human, mouse, and rat postnatal bone marrow primitive, multipotent adult progenitor cells (MAPCs) that can differentiate into most mesodermal cells and neuroectodermal cells in vitro and into all embryonic lineages in vivo. Here, we show that MAPCs can also differentiate into hepatocyte-like cells in vitro. Human, mouse, and rat MAPCs, cultured on Matrigel with FGF-4 and HGF, differentiated into epithelioid cells that expressed hepatocyte nuclear factor$3 \beta$ (HNF-3 $\beta$ ), GATA4, cytokeratin 19 (CK19), transthyretin, and $\alpha$-fetoprotein by day 7 , and expressed CK18, HNF-4, and HNF-1 $\alpha$ on days 14-28. Virtually all human, as well as a majority of rodent cells stained positive for albumin and CK18 on day $21 ; 5 \%$ (rodent) to $25 \%$ (human) cells were binucleated by day 21 . These cells also acquired functional characteristics of hepatocytes: they secreted urea and albumin, had phenobarbital-inducible cytochrome $\mathrm{p} 450$, could take up LDL, and stored glycogen. MAPCs, which can be expanded in vitro and maintained in an undifferentiated state for more than 100 population doublings, can thus differentiate into cells with morphological, phenotypic, and functional characteristics of hepatocytes. MAPCs may therefore be an ideal cell for in vivo therapies for liver disorders or for use in bioartificial liver devices.
\end{abstract}

J. Clin. Invest. 109:1291-1302 (2002). doi:10.1172/JCI200215182.

\section{Introduction}

During embryogenesis, the first sign of liver morphogenesis is a thickening of the ventral endodermal epithelium, which occurs between embryonic day (e) 7.5 and e8.5 in the mouse (1). Little is known about the signals involved in initial endoderm formation and subsequent endoderm specification. Early in gastrulation (e6-e7) endoderm is not specified, not even in an anterior/posterior direction (2). However, recent studies showed that ex vivo exposure of endoderm to FGF-4 posteriorizes the early endoderm, which is now competent to express hepatic markers (3). By e8.5 in the mouse, definitive endoderm has formed the gut tube and expresses hepatocyte nuclear factor- $3 \beta$ $(\mathrm{HNF}-3 \beta)$ (4). The foregut endoderm is induced to the hepatocyte lineage by acidic FGF (aFGF) and bFGF, both produced by the adjacent cardiac mesoderm (1), which are required to induce a hepatic fate and not the default pancreatic fate (1). Basic morphogenetic proteins (BMPs) produced by the transversum mesenchyme are also required as they increase levels of the GATA4 transcription factor that promote the ability of endoderm to respond to FGFs (1). GATA4 and HNF- $3 \beta$ are required for hepatic specification and are important effectors of downstream events leading to hepatocyte differentiation because they upregulate markers of hepatocyte specific expression such as albumin, among others.

In most instances, mature hepatocytes can undergo several cell divisions and are responsible for hepatic cell replacement. As a result, there has been great controversy about the existence and function of a liver stem cell. During extensive liver necrosis due to chemical injury or when hepatocytes are treated with chemicals that block their proliferation, a population of smaller cells with oval shape, termed oval cells, emerges and proliferates (5). These oval cells may constitute the "stem cell" compartment in the liver. Oval cells reside in the smallest units of the biliary tree, called the canals of Hering, from where they migrate into the liver parenchyma (6). Oval cells are bipotential, giving rise in vitro and in vivo to both hepatocytes and bile ductular epithelium. Oval cells express several hematopoietic markers such as Thy1.1, CD34, Flt3-receptor, and c-Kit and also express $\alpha \mathrm{FP}$, cytokeratin 19 (CK19), $\gamma$-glutamyl-transferase, and OV-6. The origin of oval cells is not known $(5,7,8)$.

Until recently, it was believed that hepatocytes could only be derived from cells of endodermal origin and their progenitors. However, recent studies 
suggest that nonendodermal cells may also form hepatocytes in vivo and in vitro (8-13). Following bone marrow (BM) transplantation, oval cells are derived from the donor BM (8). Transplantation of enriched hematopoietic stem cells (HSCs) in $\mathrm{FAH}^{-/-}$ mice, an animal model of tyrosenimia type I, resulted in the proliferation of large numbers of donor Lac $Z^{+}$hepatocytes, and animals had restored biochemical function of the liver (13). Furthermore, single HSCs may not only repopulate the hematopoietic system but also contribute to epithelium of lung, skin, liver, and gut (10). Exocrine pancreatic tumor cells treated in vitro with dexamethasone (Dex) with or without oncostatin M (OSM) may acquire a hepatocyte phenotype (14). Finally, mouse embryonic stem (ES) cells spontaneously acquire a hepatocyte phenotype, a process that is enhanced by treatment with aFGF, HGF, OSM, and Dex (15).

Recently, we demonstrated that human, mouse, or rat postnatal BM contains primitive progenitors, termed multipotent adult progenitor cells, or MAPCs, that copurify with mesenchymal stem cells (MSCs) $(16,17)$. Human and rodent MAPCs are CD44- ${ }^{-}$CD45 ${ }^{-}$, HLA class $\mathrm{I}^{-}$and $\mathrm{II}^{-}$, and $\mathrm{CKit}^{-}$. MAPCs can be expanded under defined serum-low conditions for more than 100 population doublings without telomere shortening and remain karyotypically normal. MAPCs not only differentiate into mesenchymal cell types (osteoblasts, chondroblasts, adipocytes, and skeletal myoblasts), but also into endothelium, as well as cells with neuroectodermal phenotype and function. We demonstrate here that single MAPCs not only differentiate into mesodermal and neuroectodermal cells, but also into cells with morphological, phenotypic, and functional characteristics of hepatocytes in vitro.

\section{Methods}

Chemicals and cytokines. EGF was from Sigma-Aldrich (St. Louis, Missouri, USA), leukemia inhibitory factor (LIF) was from Chemicon International (Temecula, California, USA), and PDGF-BB, FGF-4, and HGF were from R\&D Systems Inc. (Minneapolis, Minnesota, USA). OSM, aFGF, basic FGF (bFGF), and keratinocyte growth factor (KGF) were from Research Diagnostics Inc. (Flanders, New Jersey, USA). DMSO and sodium butyrate were from Sigma-Aldrich.

MAPC isolation and culture. BM was obtained from C57BL/6 mice or Sprague Dawley rats. For human MAPCs (hMAPCs), BM was obtained from ten healthy donors (2-55 years) with informed consent, according to guidelines from the University of Minnesota Committee on the Use of Human Subjects in Research. Mouse (m) MAPCs, rat (r) MAPCs, or hMAPC were selected and cultured as described (ref. 16, and Y. Jiang, unpublished observations). Cells were used for differentiation to hepatocytes after more than 50 population doublings (mMAPCs and rMAPCs) or more than 35 population doublings (hMAPCs).
Hepatocyte differentiation. The mMAPCs or rMAPCs were plated at $5 \times 10^{3}$ to $25 \times 10^{3}$ cells $/ \mathrm{cm}^{2}$ on $0.01-10$ $\mu \mathrm{g} / \mathrm{ml}$ fibronectin ( $\mathrm{FN}), 0.01-8 \mu \mathrm{g} / \mathrm{ml}$ collagen (SigmaAldrich), or 1\% Matrigel (Becton-Dickinson and Co., Franklin Lakes, New Jersey, USA) in serum-free medium (60\% low glucose DMEM) (DMEM-LG; Life Technologies Inc., Grand Island, New York, USA), 40\% MCDB-201 (Sigma-Aldrich), supplemented with 1× insulin/transferrin/selenium, $4.7 \mu \mathrm{g} / \mathrm{ml}$ linoleic acid, $1 \mathrm{mg} / \mathrm{ml} \mathrm{BSA}, 10^{-8} \mathrm{M}$ Dex, $10^{-4} \mathrm{M}$ ascorbic acid 2-phosphate (all from Sigma-Aldrich), $100 \mathrm{U} / \mathrm{ml}$ penicillin, 100/ml U streptomycin (Life Technologies Inc.), with 2\% FCS (Hyclone Laboratories, Logan, Utah, USA) and $10 \mathrm{ng} / \mathrm{ml}$ each EGF (Sigma-Aldrich), LIF (Chemicon International, Temecula, California, USA), and PDGF-BB (R\&D Systems Inc.). The hMAPCs were plated at $15 \times 10^{3}$ to $30 \times 10^{3}$ cells $/ \mathrm{cm}^{2}$ on $0.1 \mu \mathrm{g} / \mathrm{ml}$ FN or $1 \%$ Matrigel in the same medium without LIF (M. Reyes, unpublished observations). After 8-12 hours, media were removed, cells washed twice with PBS) (Fischer Scientific Co., Pittsburgh, Pennsylvania, USA) and cultured in serum-free medium supplemented with 5-50 ng/ml HGF, aFGF, bFGF, FGF-4, FGF-7, or OSM, or $10 \mathrm{mg} / \mathrm{ml}$ DMSO, or $0.1-1 \mathrm{mM}$ sodium butyrate. FGF-4 and HGF were from R\&D Systems Inc. OSM, aFGF, bFGF, and FGF-7 were from Research Diagnostics Inc. DMSO and sodium butyrate were from Sigma-Aldrich.

Hepatocyte isolation and culture. Hepatocytes were harvested from 4- to 6-week-old male Sprague-Dawley rats as described (18). Hepatocyte viability after the harvest ranged from $90-95 \%$. Hepatocytes were cultured as described $(19,20)$. To form a monolayer, hepatocytes were cultured on $35 \mathrm{mM}$ Fischer culture plates (Fischer Scientific Co.) coated with $8 \mu \mathrm{g} / \mathrm{cm}^{2}$ collagen (Cohesion Technologies Inc., Palo Alto, California, USA). To form spheroids, hepatocytes were cultured on $35-\mathrm{mm}$ Primaria dishes (Becton Dickinson and Co.). In both conditions, seeding density was $5 \times 10^{4}$ cells $/ \mathrm{cm}^{2}$. Twelve hours after initial plating, medium was changed to remove dead and unattached cells. Medium was replaced every 48 hours thereafter and tested for both urea and albumin production and used as standard rat hepatocyte values.

Total RNA isolation and quantitative RT-PCR. RNA was extracted from $3 \times 10^{5}$ MAPCs or MAPCs induced to differentiate to hepatocytes. The mRNA was reverse transcribed and cDNA was amplified as follows: 40 cycles of a two-step PCR $\left(95^{\circ} \mathrm{C}\right.$ for 15 seconds, $60^{\circ} \mathrm{C}$ for 60 seconds) after initial denaturation $\left(95^{\circ} \mathrm{C}\right.$ for 10 minutes) with $2 \mu$ l DNA solution, $1 \times$ TaqMan SYBR green universal mix PCR reaction buffer using a ABI PRISM 7700 (Perkin Elmer Applied Biosystems, Boston, Massachusetts, USA). Primers used for amplification are listed in Table 1. The mRNA levels were normalized using $\beta$-actin (mouse and human) or $18 \mathrm{~S}$ (rat) as housekeeping genes and compared with mRNA levels in freshly isolated rat or mouse hepatocytes, rat hepatocytes cultured for 7 days, or mRNA 
from human adult liver RNA purchased from CLONTECH Laboratories (Palo Alto, California, USA).

Western blot analysis. Western blot analyses were performed as described (16). Ab's to $\alpha F P$, human albumin, and CK18 were diluted 1:1,000 in blocking buffer. Goat anti- $\beta$-actin $(1: 1,000)$ was from Santa Cruz Biotechnology Inc. (Santa Cruz, California, USA). Secondary Ab's were horseradish peroxidase-linked (HRP-linked) goat anti-mouse and HRP-linked donkey anti-goat (Amersham Life Sciences Inc., Arlington Heights, Illinois, USA). Enhanced chemiluminescence was performed according to manufacturer's instructions (Amersham Life Sciences Inc.).

Immunofluorescence. Undifferentiated and differentiated MAPCs were fixed with $4 \%$ paraformaldehyde (Sigma-Aldrich) for 4 minutes at room temperature. For cytoskeletal proteins, samples were fixed with methanol (Sigma-Aldrich) for 2 minutes at $-20^{\circ} \mathrm{C}$. For nuclear ligands, cells were permeabilized with 0.1\% Triton-X 100 (Sigma-Aldrich) for 10 minutes. Slides were incubated sequentially for 30 minutes each with primary $\mathrm{Ab}$, and FITC, phycoerythrin, $\mathrm{Cy} 3$ or Cy5-coupled anti-mouse, anti-goat, or anti-rabbit $\operatorname{IgG~Ab.~Between~each~step,~slides~were~washed~with~}$ PBS $\pm 1 \%$ BSA. Ab's against HNF-3 $\beta$, HNF- $1 \alpha$, GATA4, and c-met (Santa Cruz Biotechnology Inc.) were used at a 1:50 dilution. Ab's against human albumin (rabbit polyclonal, 1:1,000, or mouse monoclonal clone HAS-11, 1:1,000), human $\alpha \mathrm{FP}$ (clone C3, 1:500), pan-CK (clone C-11, 1:400), CK18 (clone CY-90, 1:800), and CK8 (clone M20, 1:200) were from Sigma-Aldrich. Ab against CD26 (1:200) was from Neomarkers Inc. (Fremont, California, USA). Ab's against mouse albumin (1:500) were from Accurate Chemical \& Scientific Corp. (Westbury, New York, USA), whereas Ab's against mouse $\alpha F P(1: 250)$ and human carcinoembryonic antigen (CEA) (1:400) were from DAKO Corp. (Carpinteria, California, USA). $\mathrm{Ab}$ 's against rat albumin (1:500) was from Cappel (Turnhout, Belgium). Secondary Ab's anti-goat IgGCy-3 (1:40), anti-goat IgG-FITC (1:160), anti-mouse IgG-Cy-3 (1:150), anti-mouse IgG-FITC (1:320), anti-rabbit-FITC (1:160), and anti-rabbit Cy-3 (1:30) were from Sigma-Aldrich. Secondary Ab's anti-goat IgG-Cy-5 (1:200), anti-mouse IgG-Cy-5 (1:200), and anti-rabbit Cy-3 (1:200) were from Jackson ImmunoResearch Laboratories Inc. (West Grove, Pennsylvania, USA). TOPRO-3 (1:500) was from Molecular Probes Inc. (Eugene, Oregon, USA). Cells were examined by fluorescence microscopy using a Zeiss Axiovert scope (Carl Zeiss Inc., Thornwood, New York, USA) as well as by confocal fluorescence microscopy using a confocal 1024 microscope (Olympus AX70; Olympus Optical Co., Tokyo, Japan). To enumerate the number of cells staining positive for a given marker, we evaluated $200-300$ cells per culture under low power and enumerated the total number of cells staining positive with a given $\mathrm{Ab}$.
Urea assay. Urea concentrations were determined by colorimetric assay (640-1; Sigma-Aldrich) per manufacturer's instructions. Rat hepatocytes grown in monolayer and fetal mouse liver buds were used as positive controls and culture medium used as negative control. The assay can detect urea concentrations

\section{Table 1}

Primers used

Primer name
Mouse

HNF-1 $\alpha$ S: 5'-TTCTAAGCTGAGCCAGCTGCAGACG-3'

HNF-3 $\beta$ A: 5'-GCTGAGGTTCTCCGGCTCTITCAGA-3' S: 5'-CCAACATAGGATCAGATG-3'
A: 5'-ACTGGAGCAGCTACTACG-3'

GATA4

CK18 S: 5'-AGGCATTACATACAGGCTCACC-3' A: 5'-CTGTGGCCTCTATCACAAGATG-3' S: 5'-TGGTACTCTCCTCAATCTGCTG-3' A: 5'-CTCTGGATTGACTGTGGAAGTG-3' S: 5'-CATGGTTCTTCTTCAGGTAGGC-3' A: 5'-GCTGCAGATGACTTCAGAACC-3'

CK19 S: 5'-TCAACTGTCAGAGCAGAGAAGC-3' A: 5'-AGACTGCCTTGTGTGGAAGACT-3' S: 5'-GTGAAACAGACTTCCTGGTCCT-3' A: $5^{\prime}$-GCCCTACAGACCATGAAACAAG-3' S: 5'-TCTCTCAATTCTGGGGGTTG-3' A: 5'-TTTCACAGCCAACGACTCTG-3' S: 5'-GATGATGTTGGCTGTGATGC-3' A: 5'-CTGGCCACCATGAAAGAGTT-3' Cyp2b9 Cyp2b13 S: 5'-CTGCATCAGTGTATGGCATTाT-3' A: 5'-TTTGCTGGAACTGAGACTACCA-3'

Human $\alpha F P$

S: 5'-TGCAGCCAAAGTGAAGAGGGAAGA-3' A: $5^{\prime}$-CATAGCGAGCAGCCCAAAGAAGAA-3'

Albumin S: 5'-TGC TTG AATGTGCTGATGACAGGG-3' A: 5'-AAGGCAAGTCAGCAGGCATCTCATC-3' S: 5'-ATGGCCGAGCAGAACCGGAA-3' A: 5'-CCATGAGCCGCTGGTACTCC-3' S: 5'-TGGTACTCTCCTCAATCTGCTG-3' A: 5'-CTCTGGATTGACTGTGGAAGT-3'

CYP1B1 S: 5'-GAGAACGTACCGGCCACTATCACT-3'

CYP2B6 A: 5'-GTTAGGCCACTTCAGTGGGTCATGAT-3' S: 5'-GATCACACCATATCCCCGGA-3' A: 5'-CACCCTACCACCCATGACCG-3'

Rat HNF-1 $\alpha$ S: 5'-AGCTGCTCCTCCATCATCAGA-3' HNF-3 $\beta$ A: 5'-TGTTCCAAGCATTAAGTITTCTATTCTAA-3' S: 5'-CCTACTCGTACATCTCGCTCATCA-3' A: 5'-CGCTCAGCGTCAGCATCTT-3' S: 5'-GCCCTGGACTCCAGCAACT-3' A: 5'-ACTTTGCCATCCACGACCTT-3' S: 5'-ACCATGCAGAACCTGAACGAT-3' A: 5'-CACCTCCAGCTCGCCATTAG-3' Albumin S: $5^{\prime}$-CTGGGAGTGTGCAGATATCAGAGT-3' A: 5'-GAGAAGGTCACCAAGTGCTGTAGT-3' $\alpha F P \quad$ S: $5^{\prime}$-GTCCTTTCTTCCTCCTGGAGAT-3' A: 5'-CTGTCACTGCTGATTTCTCTGG-3' S: 5'-CAGCAGTGGTGCTGTAGGAGTA-3' A: 5'-GGGTAGAACTGGACACCAAATC-3' CYP2B1 S: 5'-GAGTTCTTCTCTGGGTTCCTG-3' A: 5'-ACTGTGGGTCATGGAGAGCTG-3' 
Table 2

Optimization of MAPC differentiation into hepatocyte-like cells

\begin{tabular}{|c|c|c|c|c|c|c|}
\hline \multirow[b]{2}{*}{$\mathrm{FN}$} & \multicolumn{3}{|c|}{ Mouse and rat } & \multicolumn{3}{|c|}{ Human } \\
\hline & FGF-4 & HGF & FGF-4 + HGF & FGF-4 & HGF & FGF-4 + HGF \\
\hline Albumin & $++/++$ & $++/+$ & $++/++$ & +++++ & +++++ & +++++ \\
\hline CK18 & $++/++$ & $++/+$ & $+++/++$ & +++++ & +++++ & +++++ \\
\hline HNF-3 $\beta$ & $+++/+++$ & $+++/+$ & $++++/+++$ & +++++ & NT & +++++ \\
\hline \multicolumn{7}{|l|}{ Matrigel } \\
\hline Albumin & $++/++$ & $+/+$ & $+++/+++$ & +++++ & NT & +++++ \\
\hline CK18 & $++/++$ & $++/+$ & $+++/+++$ & +++++ & NT & +++++ \\
\hline HNF-3 $\beta$ & $+++/+++$ & $+++/++$ & $++++/++++$ & +++++ & NT & +++++ \\
\hline \multicolumn{7}{|l|}{ Collagen } \\
\hline Albumin & - & - & - & NT & NT & NT \\
\hline CK18 & - & - & - & NT & NT & NT \\
\hline HNF-3 $\beta$ & - & - & - & NT & NT & NT \\
\hline
\end{tabular}

Mouse and rat: $\operatorname{mMAPCs}(n=7)$ and rMAPCs $(n=5)$ were cultured at $21.5 \times 10^{3}$ cells $/ \mathrm{cm}^{2}$ on FN, Matrigel, and collagen with the respective cytokines for 14 days. Human: hMAPCs $(n=3)$ were cultured at $3 \times 10^{4} \mathrm{cells} / \mathrm{cm}^{2}$ on FN and Matrigel with the respective cytokines for 28 days. Samples were evaluated by immunohistochemical staining of albumin, CK18, and HNF-3ß. -, 0\%; +, 20\%; ++, 30\%; +++, $40 \%$; ++++, 60\%; +++++, $80 \%$ cells staining positive for specific markers. NT, not tested.

as low as $10 \mathrm{mg} / \mathrm{ml}$. Because the assay also measures ammonia, samples were assessed before and after urease addition. No urea or ammonia was detected in culture medium alone.

Albumin ELISA. Rat albumin concentrations were determined by a competitive ELISA described previously $(20,21)$. Human and mouse albumin concentrations were determined using a similar ELISA method with substitution of human or mouse albumin and anti-human or anti-mouse albumin Ab's for the rat components where appropriate. Peroxidase-conjugated anti-human albumin and reference human albumin were from Cappel. Peroxidase-conjugated and affinity-purified anti-mouse albumin and reference mouse albumin were from Bethyl Laboratories (Montgomery, Texas, USA). To ensure specificity of the ELISA, human, mouse, and rat Ab's were incubated for 2 hours at $37^{\circ} \mathrm{C}$ with $3 \% \mathrm{BSA}$ in distilled water $\left(\mathrm{dH}_{2} \mathrm{O}\right)$. ELISAs had a sensitivity of at least $1 \mathrm{ng} / \mathrm{ml}$.

Pentoxyresorufin assay. The mMAPC-hepatocyte aggregates were formed by plating day 14 FGF-4treated and HGF-treated mMAPCs at $5 \times 10^{4}$ cells $/ \mathrm{cm}^{2}$ on non-tissue-culture plates, which were placed on a shaker at 10 revolutions per minute for 5 hours. Cell aggregates were transferred to Primaria dishes and allowed to compact for 4 days in the presence or absence of $1 \mathrm{mM}$ phenobarbital. The hMAPChepatocyte aggregates were formed by the hanging drop method. Briefly, $10^{3}$ hMAPCs treated for 24 days with FGF-4 and HGF were placed into $100 \mu \mathrm{l}$ drops with or without $1 \mathrm{mM}$ phenobarbital. After 4 days, aggregates were collected and cytochrome P450 (CYP) activity assessed by pentoxyresorufin (PROD) assay. Pentoxyresorufin (Molecular Probes Inc.) is $O$ dealkylated by CYP, changing a nonfluorescent compound into a fluorescent compound, resorufin (19). Fluorescence intensity caused by PROD metabolism consequently estimates CYP activity. Assessment and detection of resorufin in situ was performed using confocal microscopy as described (21).

Uptake of $a-L D L$. The Dil-Ac-LDL staining kit was purchased from Biomedical Technologies (Stoughton, Massachusetts, USA). The assay was performed per manufacturer's recommendations.

Periodic acid-Schiff for glycogen. Slides were oxidized in $1 \%$ periodic acid for 5 minutes and rinsed three times in $\mathrm{dH}_{2} \mathrm{O}$. Afterward, slides were treated with Schiff's reagent for 15 minutes, rinsed in $\mathrm{dH}_{2} \mathrm{O}$ for 5-10 minutes, stained with Mayer's hematoxylin for 1 minute, and rinsed in $\mathrm{dH}_{2} \mathrm{O}$.

Additional methods include culture of HUH7 cells (22), karyotyping (16), transduction of cells (16), and splinkerette flanking region PCR for the retroviral insert (Y. Jiang, unpublished observations; and $\mathrm{M}$. Reyes, unpublished observations) all done as described in references.

\section{Results}

mMAPCs, rMAPCs, and hMAPCs acquire a hepatocyte-like phenotype when cultured with FGF-4 and/or HGF. The MMAPCs, rMAPCs, and hMAPCs were selected and cultured as we described previously (16). We first determined optimal conditions for MAPC differentiation into hepatocyte-like cells. We tested the effect of different ECM components and cytokines known to induce hepatocyte differentiation in vivo or from ES cells $(1,4,14,15,23,24)$ on mMAPC or rMAPC differentiation to hepatocytes. Because differentiation requires cell cycle arrest, we also tested the effect of cell density. To demonstrate differentiation to hepatocyte like cells, we stained cells after 14 days with Ab's against albumin, CK18, and HNF3 $\beta$.

Optimal differentiation of mMAPCs or rMAPCs to albumin-, CK18-, and HNF-3 $\beta$-positive epithelioid cells was seen when MAPCs were plated at $21.5 \times 10^{3}$ 
cells $/ \mathrm{cm}^{2}$ in the presence of $10 \mathrm{ng} / \mathrm{ml} \mathrm{FGF-4} \mathrm{and} 20$ $\mathrm{ng} / \mathrm{ml} \mathrm{HGF}$ on Matrigel (Table 2). After 14 days, the percentage of albumin-, CK18-, and HNF-3 $\beta$-positive epithelioid cells was $61.4 \% \pm 4.7 \%$, and $17.3 \%$ of cells were binucleated. When plated on FN, differentiation to CK18- and HNF-3 $\beta$-positive epithelioid cells was also seen, even though only $53.1 \% \pm 6.3 \%$ of cells stained and fewer (10.9\%) binucleated cells were seen.

Culture with FGF-4 or HGF yielded albumin-, CK18-, and HNF- $3 \beta$-positive epithelioid cells, but the percentage of albumin-, CK18-, and HNF- $3 \beta$-positive cells was higher when mMAPCs or rMAPCs were treated with both FGF-4 and HGF (Table 2). Addition of aFGF, bFGF, FGF-7, BMPs, or OSM did not increase the percentage of cells positive for hepatocyte markers (not shown), while 1\% DMSO and 0.1-10 $\mathrm{mM}$ sodium butyrate did not support differentiation of mMAPCs or rMAPCs to cells positive for hepatocyte markers (not shown).

When we tested cell densities between 2.5 and $25 \times 10^{3}$ cells $/ \mathrm{cm}^{2}$, the highest percentage of cells with hepatocyte markers was seen in cultures seeded at $21.5 \times 10^{3}$ cells $/ \mathrm{cm}^{2}$. No hepatocyte differentiation was seen when cells were plated at less than $12.5 \times 10^{3}$ cells $/ \mathrm{cm}^{2}$.

The hMAPCs (Table 2) were plated at $3 \times 10^{3}$ to $30 \times 10^{3}$ cells $/ \mathrm{cm}^{2}$ on $10 \mathrm{ng} / \mathrm{ml} \mathrm{FN}$ or $1 \%$ Matrigel with aFGF, bFGF, FGF-7, 1\% DMSO, HGF, and/or FGF-4. Only cells treated with $10 \mathrm{ng} / \mathrm{ml} \mathrm{FGF-4}$ alone, 20 $\mathrm{ng} / \mathrm{ml} \mathrm{HGF}$ alone, or a combination of both differentiated into epithelioid cells that expressed albumin, CK18, and HNF-3 $\beta$. The hMAPCs plated at $15 \times 10^{3}$ to $30 \times 10^{3} \mathrm{cell} / \mathrm{cm}^{2}$ differentiated into epithelioid cells, whereas hMAPCs plated at $3 \times 10^{3} \mathrm{cell} / \mathrm{cm}^{2}$ died. Like mMAPC or rMAPC, the percentage of albumin-, CK18-, and HNF-3 $\beta$-positive epithelioid cells was higher when hMAPCs were cultured on Matrigel $(91.3 \% \pm 4.4 \%)$ than on FN $(89.5 \% \pm 5.4 \%)$, and the percentage of binucleated cells was higher on Matrigel (31.3\%) than on FN (28.7\%).

Phenotypic characterization of MAPC differentiation to hepatocyte-like cells. We further evaluated hepatocyte differentiation over time by immunofluorescence and confocal microscopy for early (HNF-3 $\beta$, GATA4, CK19, $\alpha \mathrm{FP})$ and late (CK18, albumin, HNF-1 $\alpha$ ) markers of hepatocyte differentiation. The mMAPCs or rMAPCs plated on Matrigel with FGF-4 and HGF enlarged from $8 \mu \mathrm{m}$ to $15 \mu \mathrm{m}$ diameter (Figure 1 and Table 3 ). On day $21-28$, approximately $60 \%$ of cells were epithelioid and surrounded by smaller round or spindle-shaped cells. Undifferentiated mMAPCs or rMAPCs did not express any of the liver-specific transcription factors or cytoplasmic markers. After 4 days, cells expressed HNF- $3 \beta$, GATA4, and $\alpha F P$, low levels of CK19, and very rare cells stained positive for HNF-1 $\alpha$, albumin, or CK18. At 7 days, the large epithelioid cells stained positive for HNF-3 $\beta$, GATA4, and HNF-1 $\alpha$, with increasing staining for albumin and CK18. Only rare cells expressed $\alpha$ FP. After 14, 21, and 28 days, the large epithelioid cells stained positive for GATA4, HNF-3 $\beta$, HNF-1 $\alpha$, CK18, and albumin, but not $\alpha$ FP or CK19.

The hMAPC (Figure 2 and Table 3) plated on Matrigel with FGF-4 and HGF or FGF-4 alone enlarged from 10 - to $12-\mu \mathrm{m}$ to 20 - to $30-\mu \mathrm{m}$ diameter by day 21 . After 7 days, cells expressed HNF-3 $\beta$, GATA4, and low levels of CK19, while few cells stained positive for albumin or CK18. After 14 and 21 days, more than $90 \%$ of epithelioid cells stained positive for GATA4, HNF-3 $\beta$, HNF-1 $\alpha$, HNF-4, CK18, and albumin, while only rare cells stained positive for $\alpha \mathrm{FP}$ or CK19.

Hepatocyte-like cells are derived from the same single bMAPCs that differentiated into neuroectoderm and endoderm. We showed previously that single mMAPCs or rMAPCs differentiate into endothelium, neuroectoderm, and CK18- and albumin-positive endodermal cells (Y. Jiang, unpublished observations). We also showed that single hMAPCs differentiate into mesoderm and neuroectoderm (M. Reyes, unpublished observations). Here we tested whether the same single hMAPC also differentiates into hepatocyte-like cells. As we described previously, five hMAPCs transduced with an eGFP-cDNA-containing retrovirus were added to 95 nontransduced hMAPCs and expanded to greater than $5 \times 10^{6}$ cells. Twenty percent was induced to differentiate into muscle, endothelium, neuroectoderm, and endoderm. For clone A16 used here, we showed previously using splinkerette PCR

Table 3

Immunohistochemistry pattern of hepatocyte marker expression

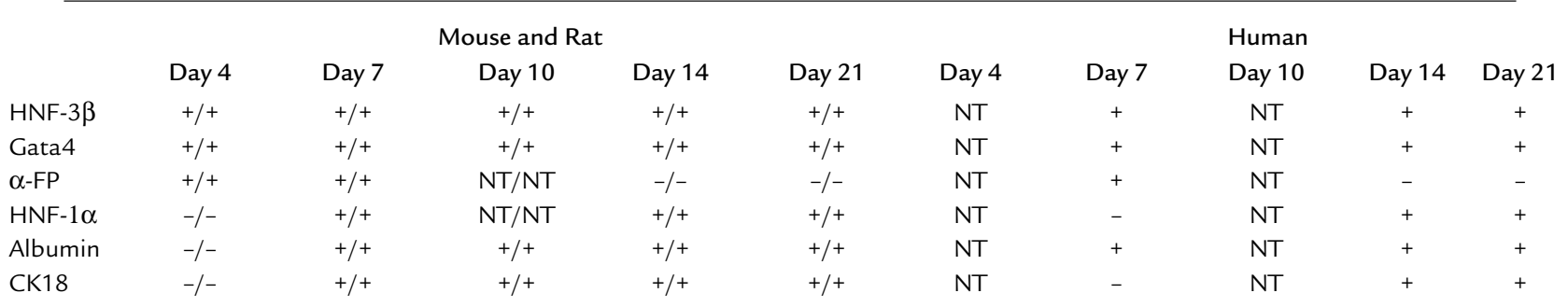

Mouse and rat: mMAPCs $(n=7)$ and rMAPCs $(n=5)$ were cultured at $21.5 \times 10^{3}$ cells $/ \mathrm{cm}^{2}$ on Matrigel with FGF-4 and HGF for $4,7,10$ and 14 days. Human: hMAPCs $(n=3)$ were cultured at $3 \times 10^{4}$ cells $/ \mathrm{cm}^{2}$ on Matrigel with the FGF- 4 for $4,7,10,14$, and 21 days. Samples were evaluated immunohistochemically for the respective markers. +, marker is expressed; -, marker is not expressed; NT, not tested. 

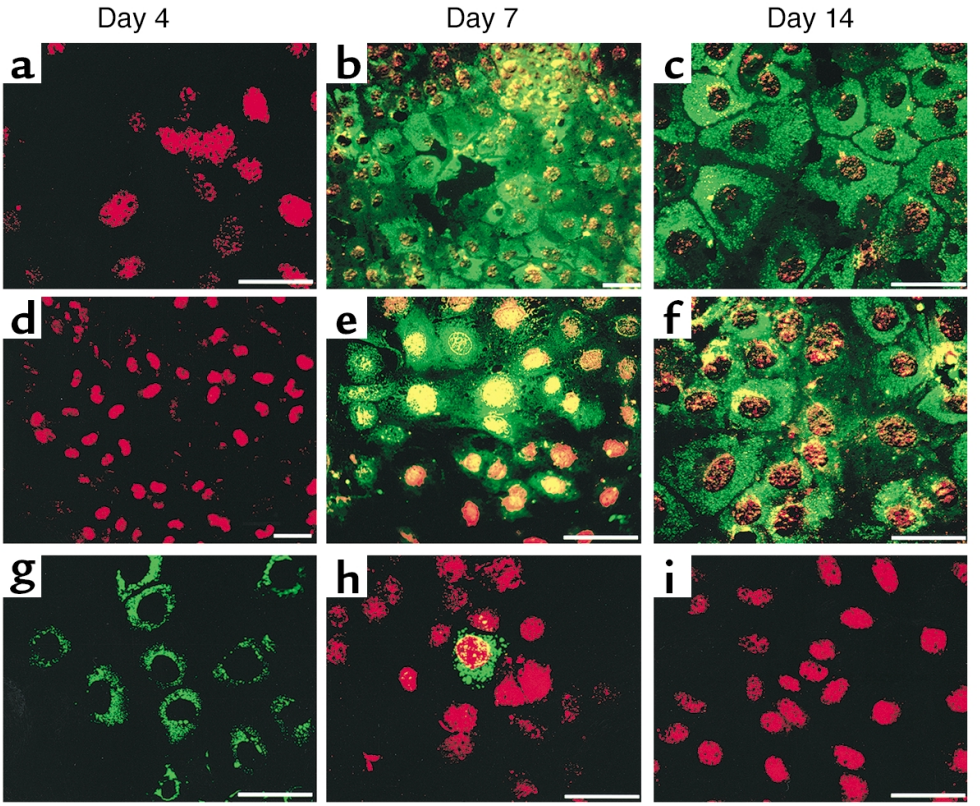

Day 14
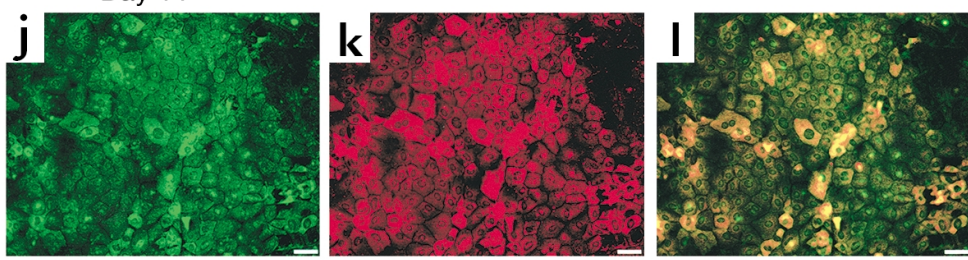

Figure 1

Phenotypic characterization of mMAPC differentiation to hepatocyte-like cells. The mMAPCs were cultured on Matrigel at $21.5 \times 10^{3}$ cells $/ \mathrm{cm}^{2}$ with FGF-4 and HGF for 4-14 days. Immunohistochemical localization of (a-c) CK18-FITC and HNF-3 $\beta-C y 3$, (d-f) albumin-FITC and GATA4-Cy3, (g-i) $\alpha$ FP-FITC and HNF- $1 \alpha-C y 3$, on day 4,7 , and 14, and (j-I) CK18-FITC and albumin-Cy3 on day 14. Scale bar, $20 \mu \mathrm{m}$. Representative example of seven experiments.

that a single retroviral insertion site was present in undifferentiated MAPCs as well as mesodermal and neuroectodermal differentiated cells. Shown in Figure 3 , $\mathrm{eGFP}^{+}$clone A16 MAPCs differentiated into CK18and albumin-positive cells. We also found that the same insertion site was present in FGF-4-treated MAPCs generated from the same cell population $\left(5^{\prime}-\right.$ TAG CGGCCGCTTGAATTCGAACGCGAGACTACTGTGACT CACACT-3', chromosome 7), proving that single hMAPCs differentiate into endoderm aside from mesoderm and neuroectoderm (M. Reyes, unpublished observations).

Quantitative RT-PCR demonstrates that FGF-4 and HGF induces hepatocyte specification and differentiation. We confirmed hepatocyte differentiation by quantitative RT-PCR for early (HNF- $3 \beta$, GATA4, CK19, $\alpha$ FP) and late (CK18, albumin, HNF-1 $\alpha$, CYP) markers of hepatocyte differentiation. On day 0, mMAPCs and rMAPCs expressed low levels of albumin, $\alpha \mathrm{FP}, \mathrm{CK} 18$, CK19, transthyretin (TTR), HNF-3 $\beta$, HNF- $1 \alpha$, and GATA4 mRNA, but no Cyp2b9 and Cyp2b13 (mouse) or CYP2B1 (rat) mRNA (Figure 4a; rat data not shown). Following treatment of mMAPCs or rMAPCs with FGF-4 and HGF, expression of HNF-3 $\beta$ and GATA4 mRNA increased on day 2 , became maximal by day 4 , decreasing slightly and leveling off by day 14 . The mRNA for $\alpha \mathrm{FP}$ and CK19 increased after day 2 and became maximal by day 4 and decreased thereafter. TTR mRNA increased after day 4 , was maximal by day 7 , and leveled off. CK18, albumin, HNF1 $\alpha$, and CYP enzyme mRNA increased after day 7 and was maximal on day 14. Between day 14 and day 21, FGF-4- and HGF-induced MAPCs expressed albumin, TTR, CK18, Cyp2b9, and Cyp2b13 (mouse) and CYP2B1 (rat) mRNA.

Undifferentiated hMAPCs expressed low levels of albumin, CK18, and CK19, CYP1B1, but not $\alpha F P$ (Figure $4 b$ ) and CYP2B6 mRNA (not shown). Levels of albumin, CK18, CK19, and CYP1B1 mRNA increased significantly in hMAPCs cultured with FGF-4 alone or with FGF-4 and HGF for 14 days compared with day 0 (MAPC) cultures. Levels of albumin, CK18, and CYP1B1 mRNA continued to increase and were higher on day 28. Although, CYP1B1 is not a specific hepatocyte marker; its upregulation suggests hepatocyte commitment and maturation. Low levels of CYP2B6 representing $0.5-1.0 \%$ of fresh liver mRNAs could be seen on day 14 and day 21 , but not day 0 (not shown). The mRNA levels of immature hepatocyte markers (CK19 and $\alpha \mathrm{FP})$ decreased as differentiation progressed and were higher in cultures induced with FGF-4 alone, whereas mRNA levels for mature hepatocytes (CK18 and albumin) were higher in FGF-4-and HGF-induced hMAPCs.

Western blot analysis demonstrates FGF-4 + HGF induces hepatocyte specification and differentiation. We also confirmed expression of hepatocyte-specific genes by Western blot analysis. Undifferentiated hMAPCs did not express CK18, albumin, or $\alpha \mathrm{FP}$ protein (Figure 4c). After treatment for 35 days with FGF-4 alone or FGF-4 and HGF, hMAPCs expressed albumin and CK18, but not $\alpha \mathrm{FP}$, consistent with the histochemi$\mathrm{cal}$ analysis shown in Figure 1b.

mMAPCs, rMAPCs, and hMAPCs acquire hepatocyte functional activity. We used six different assays to determine whether cells with morphologic and phenotypic characteristics of hepatocytes also had functional hepatocyte attributes. Urea production and secretion by hepatocyte-like cells was measured at various time points throughout differentiation. Undifferentiated MAPCs did not produce urea. Following treatment with FGF-4 and HGF, urea 

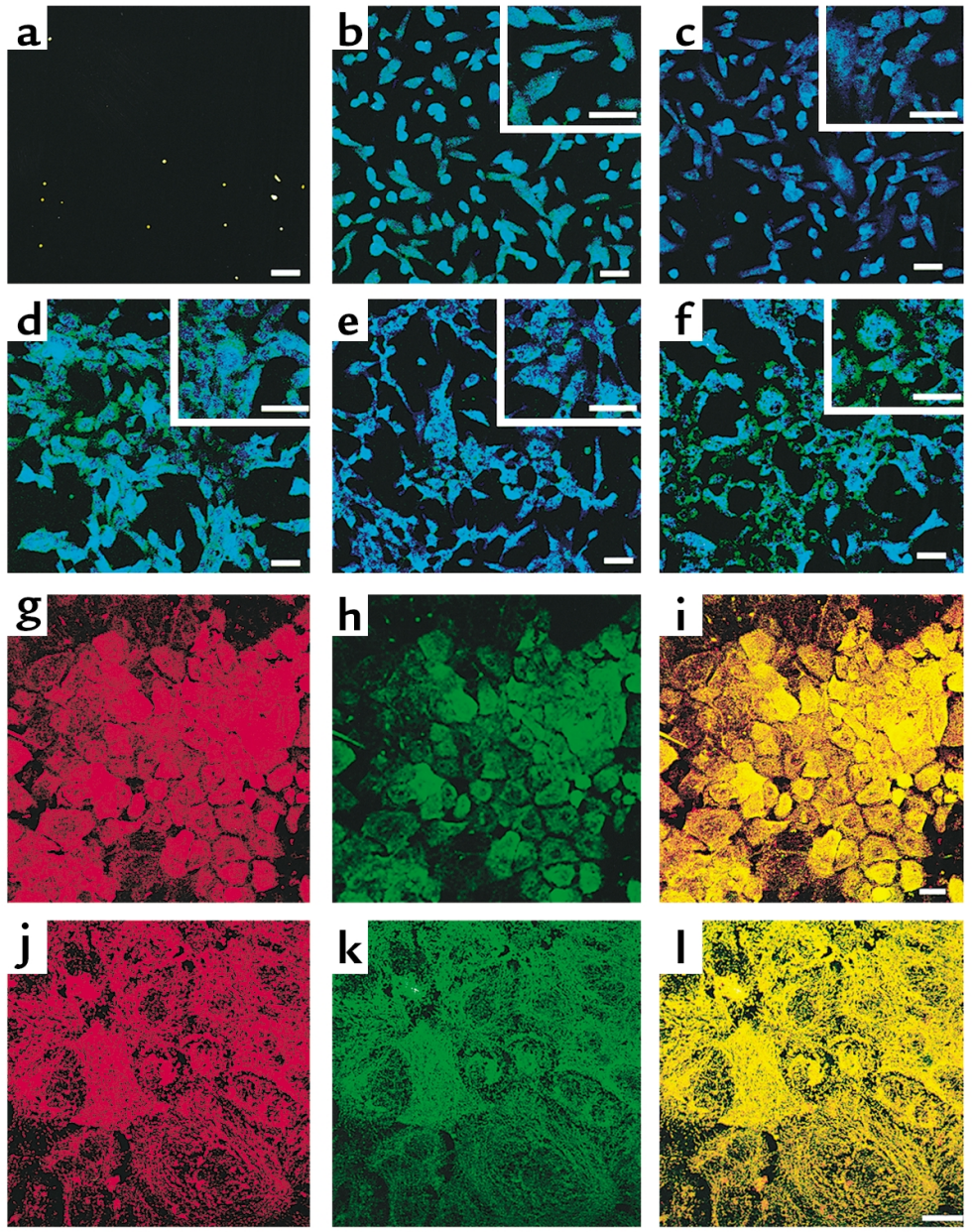

\section{Figure 2}

Phenotypic characterization of hMAPC differentiation to hepatocyte-like cells. The hMAPCs were cultured on Matrigel at $3 \times 10^{4}$ cells $/ \mathrm{cm}^{2}$ with FGF- 4 for 21 days. Representative example of three experiments. Scale bar, $25 \mu \mathrm{m}$. (a and d) Day 0 and 14, stained with albumin-FITC and HNF- $1 \alpha-C y 5$ Ab's. (b and e) Day 7 and 14, stained with CK18-FITC and GATA4-Cy5 Ab's. (c and f) Day 7 and 14, stained with CK8-FITC and HNF-3 $\beta$-Cy 5 Ab's. (g-i) Day 21, stained with CK18-Cy3 and albumin-FITC Ab's. (g) CK18-Cy3, (h) albumin-FITC, and (i) overlay. (j-I) Day 21, stained with CK8-Cy3 and CK18-FITC Ab's. (j) CK8-Cy3, (k) CK18-FITC, and (I) overlay.

monolayer cultures of primary rat hepatocytes. For all three species, significantly more urea was produced by cells differentiated on Matrigel compared with FN (data not shown).

Albumin production was measured at various time points throughout the differentiation. Undifferentiated MAPCs did not secrete albumin. Following treatment with FGF-4 and HGF, mMAPCs, rMAPCs, and hMAPCs produced albumin in a time-dependent manner (Figure 5b). As was seen for urea production, MAPCs differentiated on Matrigel produced higher amounts of albumin than when cultured on FN (data not shown). Mouse, rat, and human cells secreted similar levels of

production by MAPCs increased in a time-dependent manner (Figure 5a). The time course for urea production in mouse and rat cultures was similar. For hMAPCs treated with FGF-4 and HGF, urea was not detected on day 4 , was similar to mouse and rat cultures by day 12 , and exceeded that in mouse or rat cultures on day 21. Levels of urea produced by MAPC-derived hepatocytes were similar to that in albumin, even though albumin was not detected in human cultures on day 3 . Levels of albumin produced by mouse, rat, and human MAPC-derived hepatocytes were similar to those seen in monolayer cultures of primary rat hepatocytes.

We next assessed CYP activity in aggregates of MAPC-derived hepatocytes and primary rat liver hepatocyte spheroids using the PROD assay. No

\section{Figure 3}

Single hMAPC (clone A16) that differentiates to mesoderm and neuroectoderm, differentiates to hepatocytelike cells when cultured with FGF-4 on Matrigel for 21 days. The hMAPCs were from clone A16, described elsewhere (M. Reyes, unpublished observations), generated from cultures in which five eGFP ${ }^{+}$hMAPCs were added to 95 nontransduced hMAPCs and expanded to more than $5 \times 10^{6}$ cells. A16-derived hMAPCs were cultured with FGF-4 on Matrigel for 14 days and stained with Ab's against CK18, albumin, and HNF-3 $\beta$. Slides were evaluated by confocal microscopy for coexpression of eGFP and hepatocyte markers. Scale bar, $25 \mu \mathrm{m}$. (a-c) FGF-4-induced MAPC evaluated for eGFP expression. (d-e) Same field as a and b stained with CK18-Cy3. $\mathrm{eGFP}^{+}$cells express CK18. (f) Same field as $\mathbf{c}$ stained for albumin-Cy3 and HNF-3 $\beta-C y 5$. eGFP+ cells express albumin and HNF-3 $\beta$.
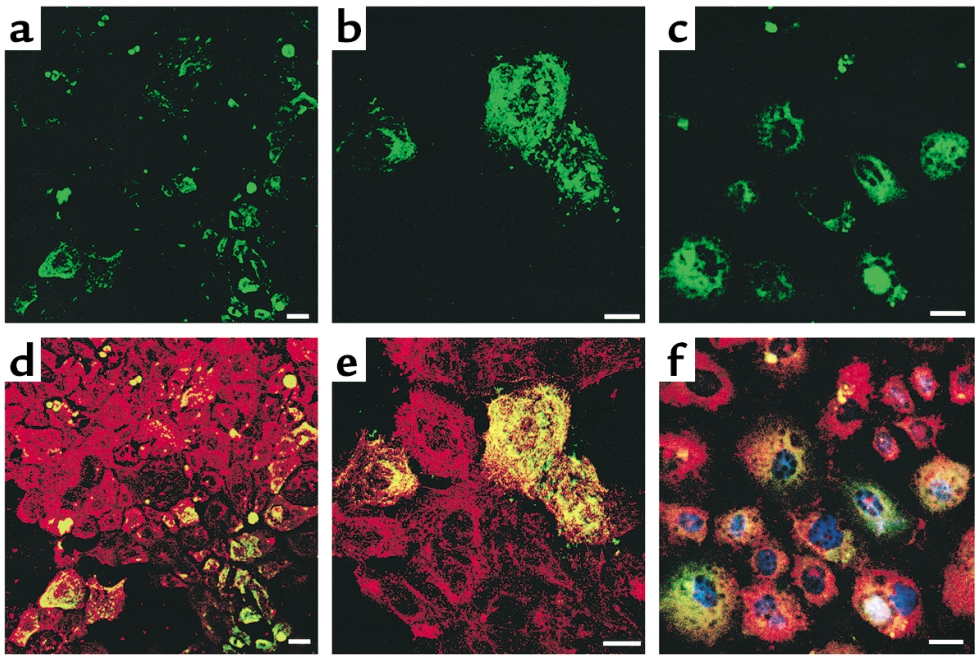


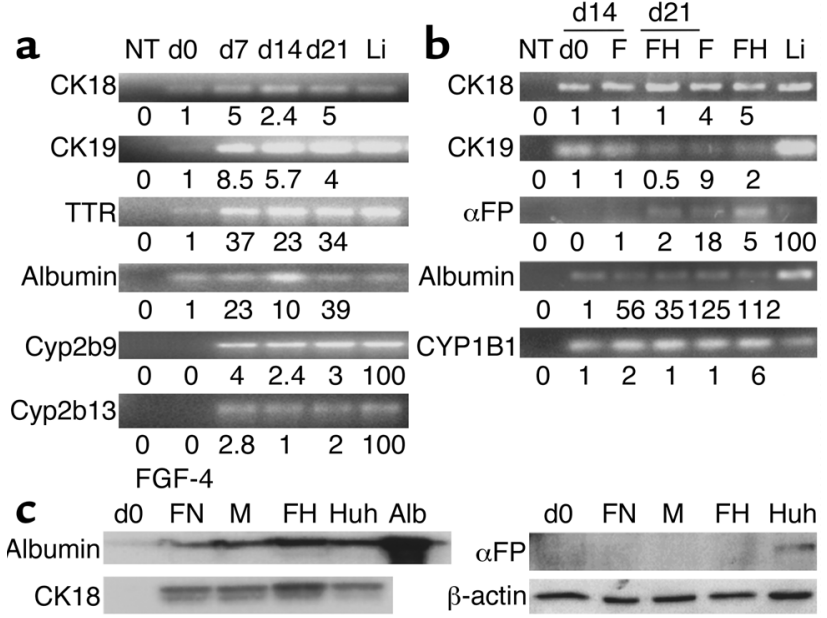

\section{Figure 4}

Quantitative RT-PCR and Western blot analyses confirm hepatocytelike phenotype. (a) mMAPCs and (b) hMAPCs were cultured on Matrigel with FGF-4 and HGF or FGF-4 alone for 21 and 28 days, respectively. At multiple time points during culture, cells were harvested and underwent quantitative RT-PCR using the SYBR green method for mRNAs as indicated. The mRNA levels were normalized using $\beta$-actin as a housekeeping gene. Numbers under the blots for CK18, CK19, TTR, albumin, and CYP1B1 represent mRNA levels after treatment relative to undifferentiated MAPCs. For $\alpha F P, C y p 2 b 9$, and Cyp2b13, numbers under the blots are relative to mRNA from the liver, because no transcripts were detected in undifferentiated MAPCs. $\mathrm{Li}$, mouse or human liver mRNA; NT, no template. Representative example of five mouse and one human study. (c) hMAPCs (from b) were cultured on Matrigel with FGF-4 and HGF or FGF-4 alone for 21 days. HUH7 cells were cultured as described. Lysates of cells were separated by SDS-PAGE, transferred to Immuno-Blot PVDF membrane, and incubated for 1 minute with Ab's against $\alpha F P, C K 18$, albumin, or $\beta$-actin. FN, FGF-4-induced hMAPCs on FN; M, FGF-4-induced hMAPCs on Matrigel; FH, FGF-4- and HGF-induced hMAPCs on Matrigel; Huh, Huh7 cell line used as control.

PROD activity was seen in aggregates of undifferentiated mMAPCs or hMAPCs. However, mMAPCs (18 days with FGF-4 and HGF) and hMAPCs (28 days, FGF-4 alone) induced to form aggregates had significant PROD activity (Figure 6). PROD activity in MAPC-derived hepatocyte aggregates was similar to that of primary rat hepatocyte aggregates. A number of different cells have CYP activity, but CYP activity upregulation by phenobarbital is seen only in hepatocytes. Therefore, we also tested CYP activity in the presence or absence of phenobarbital. Without phenobarbital, several CYP enzymes partially participate in PROD metabolism, giving an inflated fluorescence value for those samples. In contrast, in the phenobarbital-induced aggregates, PROD activity is almost wholly metabolized by mouse cytochromes Cyp2b9, Cyp2b10, and Cyp2b13, rat cytochromes CYP2B1/2 (19), and in humans, by CYP2B6. Therefore, the increased fluorescent activity is smaller than the actual increase in the protein expression of the stated CYP enzymes. When aggregates were cultured for 96 hours with phenobarbital, a $32-39 \%$ increase in
PROD activity was seen, suggesting presence of functional hepatocyte-specific Cyp2b9, Cyp2b10, and Cyp2b13 in mMAPCs and CYP2B6 in hMAPCderived hepatocytes.

We also assessed whether MAPC-derived hepatocytes can take up LDL (25) by incubating FGF-4-treated hMAPCs with LDL-Dil-acil. Cells were colabeled with either anti-CK18 or anti-Pan-CK and -HNF-3 $\beta$, or -GATA4 Ab's. After 7 days, low-level uptake of a-LDL was detected, which increased to become maximal on day 21 (Figure 7).

Another metabolic function of hepatocytes is glycogen production or gluconeogenesis. We analyzed the levels of glycogen storage by periodic acid-Schiff (PAS) staining of FGF-4- and HGF-induced mouse MAPCs and FGF-induced hMAPCs at days 3, 7, 14, and 21. Glycogen storage was first seen by day 14 , and maximum levels were seen after day 21 (Figure 8).

We also examined whether MAPC-derived hepatocytes acquire a polarized structure by labeling hMAPCs cultured for 28 days in the presence of FGF-4 with an Ab against CD26 (dipeptidypeptidase IV), an apical membrane protein (26), and an $\mathrm{Ab}$

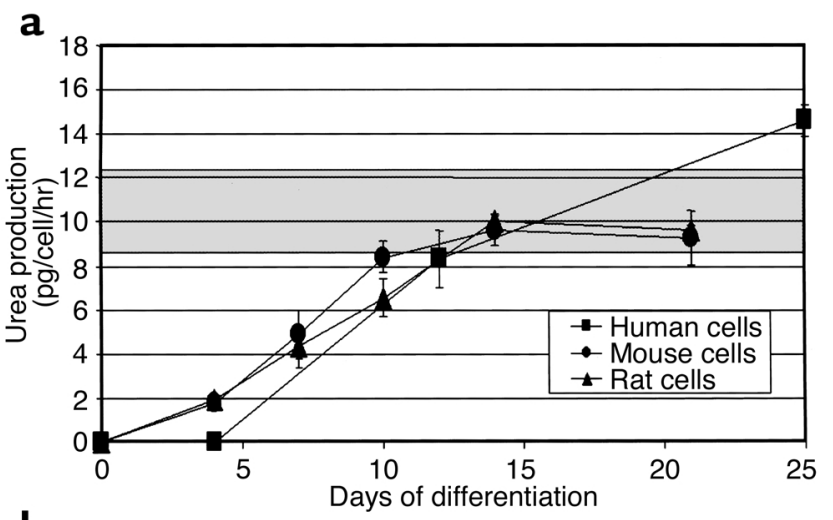

b

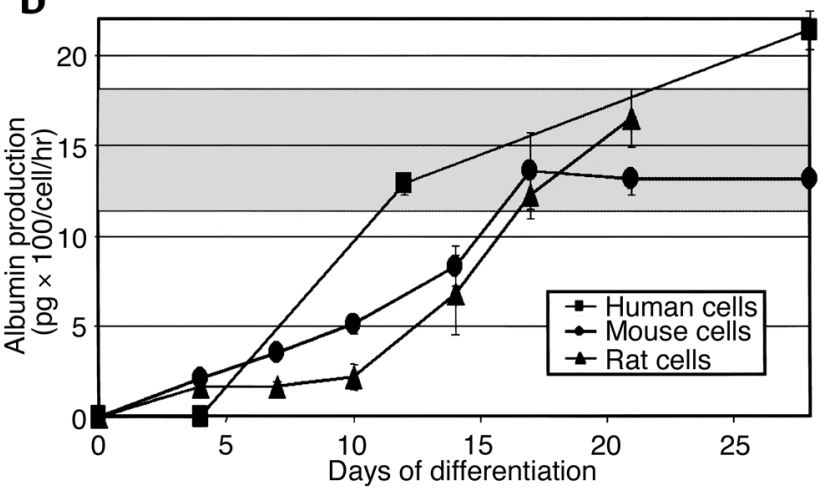

Figure 5

Urea and albumin production by mouse, rat, and human MAPCs cultured with FGF-4 and HGF on Matrigel. mMAPCs $(n=7)$, rMAPCs $(n=5)$, and hMAPCs $(n=2)$ were cultured on Matrigel with FGF-4 and HGF for 4-28 days. Gray area represents standard values for primary rat hepatocytes. (a) Urea production, expressed per cell number seeded and per hour. Studies were done in duplicate and repeated twice for each study. (b) Albumin production expressed per cell number seeded and per hour. Studies were done in duplicate and repeated twice for each study. 

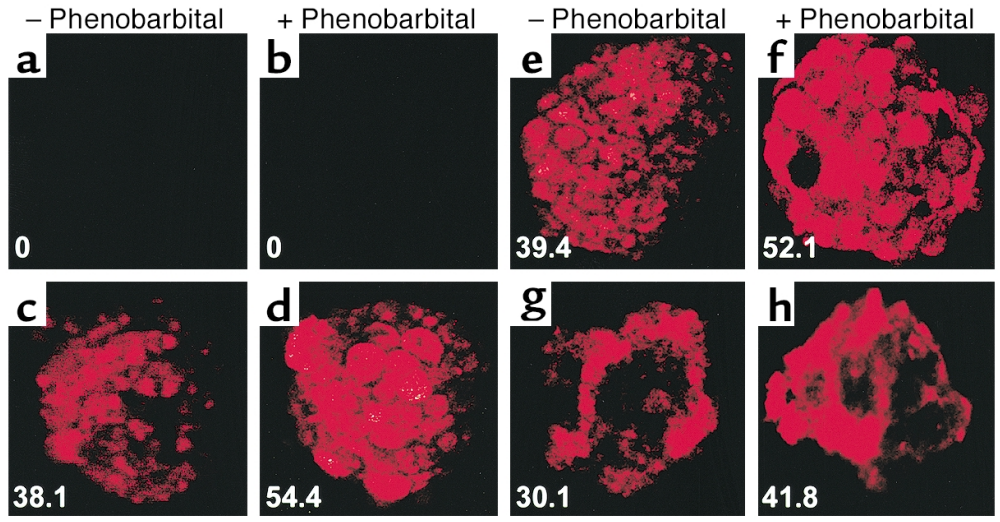

Figure 6

PROD assay on MAPC-derived hepatocytes from mice and humans. The mMAPCs and hMAPCs cultured on Matrigel with FGF-4 and HGF for 14 and 28 days were allowed to aggregate in the presence or absence of $1 \mathrm{mM}$ phenobarbital for 4 days. PROD assay was done as described in Methods. (a and $\mathbf{b}$ ) Undifferentiated mMAPC aggregates. (c and d) Primary rat hepatocytes. (e and $\mathbf{f}$ ) mMAPC-derived hepatocyte-like spheroids. ( $g$ and h) hMAPC-derived hepatocyte-like spheroids. Representative example of four mouse studies including over 120 aggregates and one human study including over 50 aggregates.

against c-met or the LDL receptor, both of which are basolateral membrane proteins $(26,27)$. We found that $6.4 \% \pm 5.6 \%$ of cells stained positive with Ab's against both apical and basolateral markers. Although the majority of cells stained in a diffuse pattern, $4.3 \% \pm 2.5 \%$ of cells expressed CD26 (Figure 9), c-met (Figure 9), and LDL receptor (not shown) in a polarized fashion. Although the frequency of cells that were polarized was low, this is consistent with what has been described for primary hepatocytes cultured in two-dimensional (2-D) cultures in vitro.

\section{Discussion}

We show that a single postnatal mouse, rat, and human BM-derived MAPC can differentiate in vitro into an endodermal cell type with hepatocyte phenotype and function. MAPCs, cultured under hepatocyte differentiation conditions and expressed in a time-dependent manner, primitive mature hepatocyte markers, shown by immunofluorescence microscopy of double- and triple-labeled cells. The protein-expression profile was hepatocyte specific and not spurious, because nonhepatocyte markers were not coexpressed with hepatocyte antigens. We confirmed results from immunohistochemistry by Western blot analysis. In addition, RT-PCR corroborated upregulation of the transcription factors HNF$3 \beta$ and GATA4, known to be important in endoderm specification and transcription factors required for subsequent hepatocyte differentiation, such as HNF$1 \alpha$, and cytoplasmic proteins such as CK19, CK18, $\alpha \mathrm{FP}$, and albumin.

To optimize hepatocyte formation, many different cytokines were tested, including aFGF, bFGF, FGF-4, HGF, FGF-7, and OSM, thought to be important for hepatocyte differentiation during embryological development. However, only FGF-4 and HGF promoted hepatocyte differentiation. FGF-4 alone induced hepatocyte differentiation. However, the degree of differentiation measured by absence of immature markers such as $\alpha \mathrm{FP}$ and CK19 was higher when cells were also treated with HGF. This is consistent with the observation that FGF-4 is important in initial endoderm patterning and may play a role in endoderm specification (23) and that HGF induces differentiation of hepatocytes that are not actively proliferating (28-31). Differentiation to cells with a hepatocyte phenotype was seen only when cells were seeded at high confluence, which may cause a postmitotic state required for differentiation in the presence of HGF. aFGF and bFGF, important in hepatocyte specification, however, did not promote hepatocyte differentiation in our system. Other signals known to influence differentiation to hepatocytes include ECM-derived signals $(32,33)$. Culture on Matrigel induced the most complete differentiation to cells expressing hepatocyte markers. Because Matrigel consists of a mixture of ECM components, we also tested whether any specific component might be responsible for the hepatocyte induction. Differentiation to cells with hepatocyte phenotype and function could also be induced when
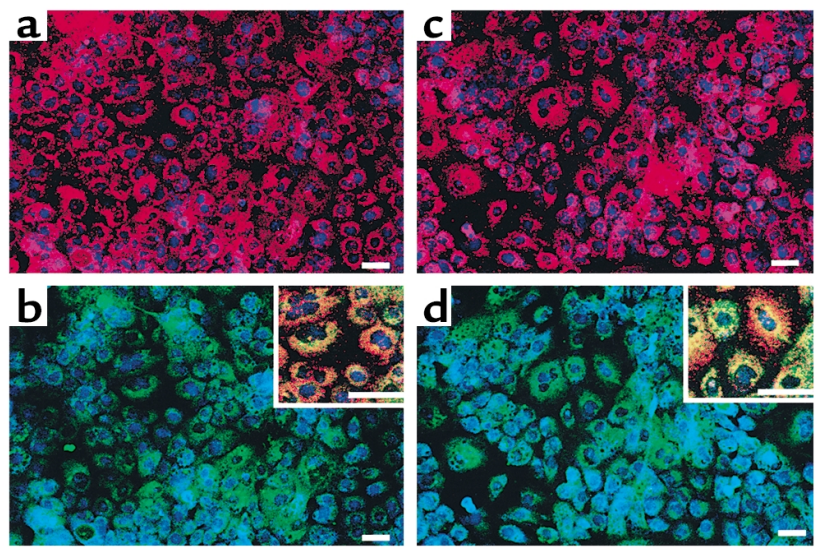

\section{Figure 7}

LDL uptake by hMAPC-derived hepatocytes. hMAPCs cultured with FGF-4 on Matrigel for 0-21 days were incubated with Dil-acil-LDL, fixed, and stained with anti-pan-CK, CK18, GATA4, and HNF-3 $\beta$ Ab's. Representative example of three studies. Scale bar, $25 \mu \mathrm{m}$. (a) Day 21, incubated with Dil-acil-LDL and stained with HNF-3 $\beta-C y 5$ Ab. (b) Same field as a stained with pan-CK-FITC and HNF-3 $\beta-C y 5$ $A b$. Inset, higher-magnification three-color view. Note the presence of binucleated cells. (c) Day 21, incubated with Dil-acil-LDL and stained with GATA4-Cy5 Ab. (d) Same field as b stained with GATA4Cy5 and CK18-FITC Ab. Inset, higher magnification showing threecolor view. Note the presence of binucleated cells. 

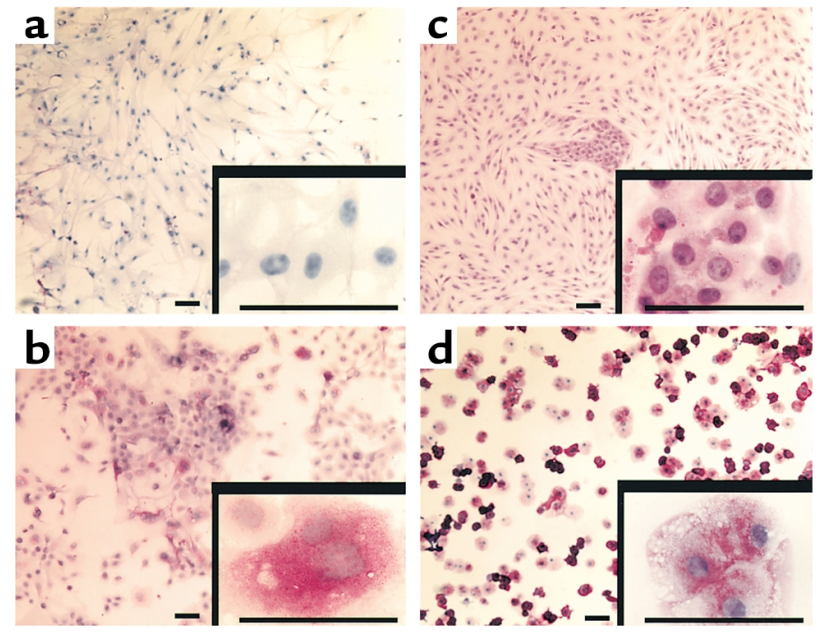

\section{Figure 8}

FGF-4-induced MAPCs produce glycogen. The rMAPCs and hMAPCs were cultured on Matrigel with FGF-4 and HGF and FGF-4 alone, respectively. Cells were fixed with methanol and stained by PAS. Glycogen storage is seen as accumulation of magenta staining. Representative example of three studies. Scale bar, 25 $\mu \mathrm{m}$. (a) Undifferentiated hMAPCs, (b) day 21 hMAPCs, (c) rMAPCs, (d) rat primary hepatocytes.

cells were cultured on FN, but not collagen I, collagen IV, or laminin (33). Culture conditions for rodent cells and human cells were similar. However, the rate with which differentiation occurred was faster for mMAPCs and rMAPCs than hMAPCs. Whether this indicates that culture conditions were not optimized for human cells, or whether this reflects differences in rate of differentiation also seen during normal embryological development, is not known.

Although we showed that FGF-4 alone or both FGF-4 and HGF induced MAPCs into cells with morphological and phenotypic characteristics of hepatocytes, this alone does not prove that cells have differentiated into hepatocytes unless one can demonstrate acquisition of functional characteristics of hepatocytes. Therefore, several functional tests were done in combination to identify functional hepatocytes. mMAPCs, rMAPCs, or hMAPCs produced urea and albumin, contained phenobarbital-inducible CYP activity, could take up Dil-acil-LDL, and contained glycogen granules. Although urea production is characteristic of hepatocyte activity, kidney tubular epithelium also produces urea (34). In contrast, albumin production is a specific test for the presence and metabolic activity of hepatocytes (34). CYP, although found in hepatocytes, is also present in many other cell types (35). However, CYP2B1 activity in rats (19), Сyp2b9 and Сyp2b13

\section{Figure 9}

in mice $(36,37)$, and CYP2B6 in humans is considered relatively hepatocyte specific. Presence of these forms of CYP was shown by RT-PCR. The specificity for hepatocytes is enhanced further when CYP activity is inducible by phenobarbital (38), as we showed. Although LDL uptake is seen in hepatocytes (25), other cells, such as endothelium, have a similar capability (39), and only hepatocytes can generate and store glycogen. Finally, structural polarity is an important property of hepatocytes in vivo. This is the result of the position of the hepatocyte between the vasculature and the biliary system and results in compartmentalization of specific tasks within the cell (40). In vivo, the apical membrane is specified to secrete bile acids and detoxified products via plasma membrane transporters, whereas the basolateral membrane is specified for exchange and secretion of plasma proteins via secretory vesicles (40). Primary hepatocytes lose this polarized structure soon after isolation and culture in 2-D in vitro (41). Furthermore, the cells adopt a flat morphology and rarely develop bile canicular structures (42). We demonstrate that a small fraction of MAPC-derived hepatocyte-like cells maintained in 2-D culture develop signs of polarization, consistent with what has been described for primary hepatocytes cultured in 2-D. When taken together, these functional tests demonstrate that MAPC from mice, rats, or humans treated in vitro with FGF-4 and HGF not only express hepatocyte markers but also have functional characteristics consistent with hepatocyte metabolic activities.

Several studies have shown that BM-derived cells may differentiate into hepatocyte-like cells in vivo and in vitro (8-13). However, most studies have not addressed the phenotype of the BM cell that differentiates into
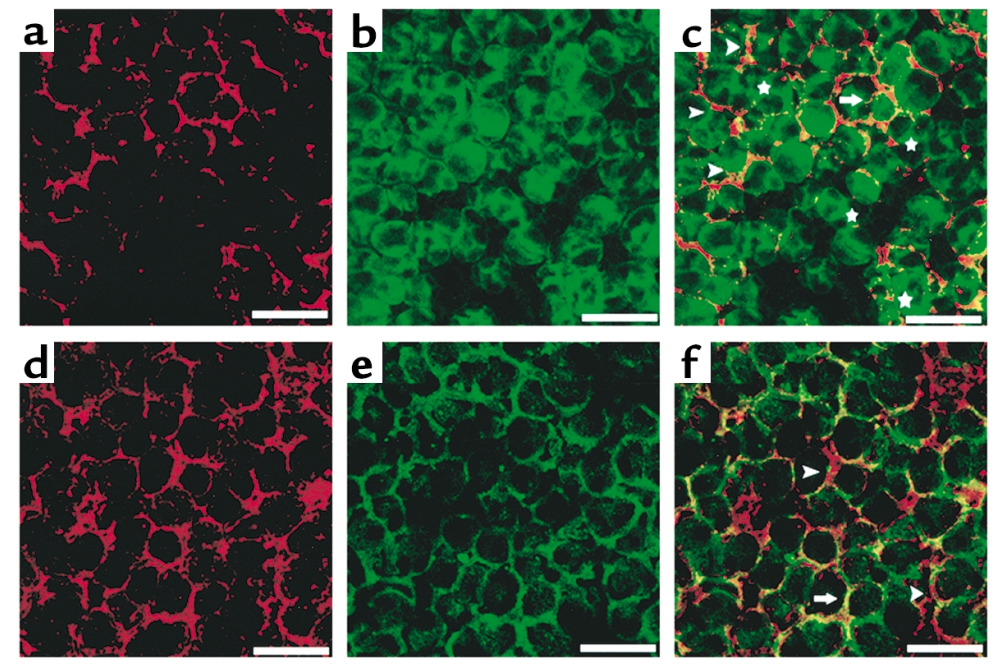

FGF-4-induced MAPCs exhibit polarization. The hMAPCs cultured with FGF-4 on Matrigel for 28 days. Representative example of two studies. Arrowhead, polarized cell; arrow, nonpolarized cell; star, binucleated cell. Scale bar, $25 \mu \mathrm{m}$. Staining for c-met-Cy3 (a), albumin-FITC (b), and overlap (c). Staining for c-met-Cy3 (d), CD26-FITC (e), and overlap (f). 
cells with hepatocyte phenotype. It is unknown whether the cells staining positive for hepatocyte markers had functional characteristics of hepatocytes and whether cells that differentiate into hepatocytes can also differentiate into mesodermal cells, such as hematopoietic cells. Lagasse et al. demonstrated that $\mathrm{cKit}^{+} \mathrm{Thy}_{1}{ }^{\text {low }} \mathrm{Sca}^{+}{ }^{+} \mathrm{Lin}^{-}$cells present in murine BM differentiate into cells with not only hepatocyte phenotype but also hepatocyte function (13). Even though such results could be seen when as few as 50 cells were transplanted, this study did not prove that the same cell that differentiates into hematopoietic cells also differentiates into hepatocytes. Krause et al. (10) showed that a single cell can repopulate the hematopoietic system and give rise to rare hepatocytes. However, no functional assessment of the hepatocytes was done. Avital et al. recently published that $\beta_{2} \mathrm{~m}^{-}$, Thy- $1^{+}$cells in mouse BM express albumin, HNF-4, C/EBP $\alpha$, and CYP3A2 mRNA and protein (43), a phenotype of hepatocyte progenitors usually found in the liver. Thus, presence of such hepatocyte progenitor cells in BM could explain the in vivo differentiation of bone marrow into hepatocytes noted in recent studies $(10,13)$.

To address the question whether cells giving rise to functional hepatocyte-like cells also give rise to other cell types, we used retroviral marking (ref. 16, and Y. Jiang, unpublished observations). We have shown recently that cells expressing albumin, CK18, and HNF- $1 \alpha$ can be generated from the same mMAPCs and rMAPCs that differentiate into cells with endothelial and neuroectodermal phenotype (Y. Jiang, unpublished observations). We here confirm that similar results are seen for hMAPCs. Extending recently published studies demonstrating derivation of cells with mesodermal and neuroectodermal phenotype and function from single hMAPCs (M. Reyes, unpublished observations), we show here that the same single hMAPC also differentiates into cells with hepatocyte morphology and phenotype. Thus, we demonstrate for the first time that MAPCs, culture-selected from bone marrow, do not express hepatocyte markers and have no functional hepatocyte activity, which, depending on the culture conditions, acquire a hepatocyte phenotype and functional characteristics of hepatocytes or phenotypic and functional characteristics of mesodermal and neuroectodermal cells. When mMAPCs are infused in normal mice, differentiation into CK18 and albumin-positive cells contributing to $3-8 \%$ of the liver parenchyma liver is seen, and mMAPCs give rise to up to $45 \%$ of hepatocytes when injected in the blastocyst (Y. Jiang, unpublished observations). We have yet to test formally whether MAPC-derived hepatocyte-like cells can rescue animals with liver failure. This has prompted us to use the term functional hepatocytelike cells rather than functional hepatocytes.

The observation that adult tissue-specific stem cells may differentiate into cells of different tissues challenges the traditional view of the biology of tissue-specific stem cells. This could be explained by the persistence of pluripotent stem cells, such as ES cells, which are capable of differentiating into many different cell types including endoderm. Alternatively, MAPCs may be the result of "dedifferentiation" of a BM-derived stem cell in our culture system, which induces chromatin remodeling and changes in cell fate determination as has been shown in the "cloning" process $(44,45)$. Irrespective of the etiology of MAPCs, the finding that MAPCs can be isolated, expanded, and maintained in vitro in an undifferentiated state for more than 100 population doublings, and induced to specifically differentiate into hepatocyte-like cells, may make the MAPC an ideal cell for in vivo therapies of genetic or acquired disorders of the liver and for use in bioartificial liver devices. Studies are ongoing to further explore the potential of MAPCs and MAPC-derived hepatocyte-like cells in the treatment of liver disorders.

\section{Acknowledgments}

This work was supported by NIH grants F31AI/ GM10291 (to M. Reyes), RO1-DK67932 and U19-DK61224 (to C.M. Verfaillie); by the Juvenile Diabetes Research Foundation, The Tulloch Family Foundation, and The McKnight Foundation (C.M. Verfaillie); and by the University of Minnesota Bone Marrow Transplant Research Fund (M. Reyes). R. Schwartz and M. Reyes are funded by the NIH Medical Scientist Training Program.

1. Zaret, K.S. 2001. Hepatocyte differentiation: from the endoderm and beyond. Curr. Opin. Genet. Dev. 11:568-574.

2. Melton, D. 1997. Signals for tissue induction and organ formation in vertebrate embryos. Harvey Lect. 93:49-64.

3. Wells, J.M., and Melton, D.A. 1999. Vertebrate endoderm development. Annu. Rev. Cell Dev. Biol. 15:393-410.

4. Zaret, K.S. 2000. Liver specification and early morphogenesis. Mech. Dev. 92:83-88.

5. Petersen, B.E. 2001. Hepatic "stem" cells: coming full circle. Blood Cells Mol. Dis. 27:590-600.

6. Theise, N.D., et al. 1999. The canals of Hering and hepatic stem cells in humans. Hepatology. 30:1425-1433.

7. Kim, T.H., Mars, W.M., Stolz, D.B., Petersen, B.E., and Michalopoulos, G.K. 1997. Extracellular matrix remodeling at the early stages of liver regeneration in the rat. Hepatology. 26:896-904.

8. Petersen, B.E., et al. 1999. Bone marrow as a potential source of hepatic oval cells. Science. 284:1168-1170.

9. Theise, N.D., et al. 2000. Derivation of hepatocytes from bone marrow cells in mice after radiation-induced myeloablation. Hepatology. 31:235-240.

10. Krause, D.S., et al. 2001. Multi-organ, multi-lineage engraftment by a single bone marrow-derived stem cell. Cell. 105:369-377.

11. Pittenger, M.F., et al. 1999. Multilineage potential of adult human mesenchymal stem cells. Science. 284:143-147.

12. Wang, X., Al-Dhalimy, M., Lagasse, E., Finegold, M., and Grompe, M. 2001 . Liver repopulation and correction of metabolic liver disease by transplanted adult mouse pancreatic cells. Am. J. Pathol. 158:571-579.

13. Lagasse, E., et al. 2000. Purified hematopoietic stem cells can differentiate into hepatocytes in vivo. Nat. Med. 6:1229-1234.

14. Shen, C.N., Slack, J.M., and Tosh, D. 2000. Molecular basis of transdifferentiation of pancreas to liver. Nat. Cell Biol. 2:879-887.

15. Hamazaki, T., et al. 2001. Hepatic maturation in differentiating embryonic stem cells in vitro. FEBS Lett. 497:15-19.

16. Reyes, M., et al. 2001. Purification and ex vivo expansion of postnatal human marrow mesodermal progenitor cells. Blood. 98:2615-2625.

17. Reyes, M., and Verfaillie, C.M. 2001. Characterization of multipotent adult progenitor cells, a subpopulation of mesenchymal stem cells. Ann. NY Acad. Sci. 938:231-235.

18. Seglen, P.O. 1976. Preparation of isolated rat liver cells. Methods Cell Biol. 13:29-83.

19. Tzanakakis, E.S., Hsiao, C.C., Matsushita, T., Remmel, R.P., and Hu, W.S. 2001. Probing enhanced cytochrome P450 2B1/2 activity in rat 
hepatocyte spheroids through confocal laser scanning microscopy. Cell Transplant. 10:329-342.

20. Tzanakakis, E.S., Hansen, L.K., and Hu, W.S. 2001. The role of actin filaments and microtubules in hepatocyte spheroid self-assembly. Cell Motil. Cytoskeleton. 48:175-189.

21. Peshwa, M.V., Wu, F.J., Follstad, B.D., Cerra, F.B., and Hu, W.S. 1994 Kinetics of hepatocyte spheroid formation. Biotechnol. Prog. 10:460-466.

22. Friedman, S.L., Shaulian, E., Littlewood, T., Resnitzky, D., and Oren, M. 1997. Resistance to p53-mediated growth arrest and apoptosis in Hep 3B hepatoma cells. Oncogene. 15:63-70.

23. Wells, J.M., and Melton, D.A. 2000. Early mouse endoderm is patterned by soluble factors from adjacent germ layers. Development 127:1563-1572.

24. Miyajima, A., et al. 2000. Role of oncostatin $M$ in hematopoiesis and liver development. Cytokine Growth Factor Rev. 11:177-183.

25. Mahley, R.W., and Ji, Z.S. 1999. Remnant lipoprotein metabolism: key pathways involving cell-surface heparan sulfate proteoglycans and apolipoprotein E. J. Lipid Res. 40:1-16.

26. Bender, V., Buschlen, S., and Cassio, D. 1998. Expression and localization of hepatocyte domain-specific plasma membrane proteins in hepatoma $\times$ fibroblast hybrids and in hepatoma dedifferentiated variants. J. Cell Sci. 111:3437-3450.

27. Koivisto, U.M., Hubbard, A.L., and Mellman, I. 2001. A novel cellular phenotype for familial hypercholesterolemia due to a defect in polarized targeting of LDL receptor. Cell. 105:575-585.

28. Oh, S.H., et al. 2000. Hepatocyte growth factor induces differentiation of adult rat bone marrow cells into a hepatocyte lineage in vitro. Biochem. Biophys. Res. Commun. 279:500-504.

29. Yoon, J.H., Lee, H.V., Lee, J.S., Park, J.B., and Kim, C.Y. 1999. Development of a non-transformed human liver cell line with differentiatedhepatocyte and urea-synthetic functions: applicable for bioartificial liver. International Journal of Artificial Organs. 22:769-777.

30. Hamamoto, R., Kamihira, M., and Iijima, S. 1999. Growth and differentiation of cultured fetal hepatocytes isolated various developmental stages. Biosci. Biotechnol. Biochem. 63:395-401.

31. Miyazaki, M., et al. 1998. Phenobarbital suppresses growth and accelerates restoration of differentiation markers of primary culture rat hepatocytes in the chemically defined hepatocyte growth medium containing hepatocyte growth factor and epidermal growth factor Exp. Cell Res. 241:445-457.
32. Mooney, D.J., Langer, R., and Ingber, D.E. 1995. Cytoskeletal filament assembly and the control of cell spreading and function by extracellular matrix. J. Cell Sci. 108:2311-2320.

33. Sanchez, A., et al. 2000. Fibronectin regulates morphology, cell organization and gene expression of rat fetal hepatocytes in primary culture. J. Hepatol. 32:242-250.

34. Dunn, J.C., Tompkins, R.G., and Yarmush, M.L. 1991. Long-term in vitro function of adult hepatocytes in a collagen sandwich configuration. Biotechnol. Prog. 7:237-245.

35. Hedlund, E., Gustafsson, J.A., and Warner, M. 2001. Cytochrome P450 in the brain; a review. Curr. Drug Metab. 2:245-263.

36. Jarukamjorn, K., Sakuma, T., Miyaura, J., and Nemoto, N. 1999. Different regulation of the expression of mouse hepatic cytochrome P450 2B enzymes by glucocorticoid and phenobarbital. Arch. Biochem. Biophys. 369:89-99.

37. Li-Masters, T., and Morgan, E.T. 2001. Effects of bacterial lipopolysaccharide on phenobarbital-induced CYP2B expression in mice. Drug Metab. Dispos. 29:252-257.

38. Zelko, I., and Negishi, M. 2000. Phenobarbital-elicited activation of nuclear receptor CAR in induction of cytochrome P450 genes. Biochem. Biophys. Res. Commun. 277:1-6.

39. Rader, D.J., and Dugi, K.A. 2000. The endothelium and lipoproteins: insights from recent cell biology and animal studies. Semin. Thromb. Hemost. 26:521-528.

40. Ihrke, G., and Hubbard, A.L. 1995. Control of vesicle traffic in hepatocytes. In Progress in liver disease. 13th edition. W.B. Saunders Co. Philadelphia, Pennsylvania, USA. 63-99.

41. Maurice, M., Rogier, E., Cassio, D., and Feldmann, G. 1988. Formation of plasma membrane domains in rat hepatocytes and hepatoma cell lines in culture. J. Cell. Sci. 90:79-92.

42. Gallin, W.J. 1997. Development and maintenance of bile canaliculi in vitro and in vivo. Microsc. Res. Tech. 39:406-412.

43. Avital, I., et al. 2001. Isolation, characterization, and transplantation of bone marrow-derived hepatocyte stem cells. Biochem. Biophys. Res. Commun. 288:156-164.

44. Wilmut, I., Schnieke, A.E., McWhir, J., Kind, A.J., and Campbell, K.H. 1997. Viable offspring derived from fetal and adult mammalian cells. Nature. 385:810-813.

45. Rideout, W.M., III, et al. 2000. Generation of mice from wild-type and targeted ES cells by nuclear cloning. Nat. Genet. 24:109-110. 